\title{
Avaliação da eficiência técnica de clones de eucalipto em escala comercial: uma abordagem empregando Data Envelopment Analysis (DEA)
}

\author{
Evaluation of the efficiency of eucalyptus clones in commercial scale: an \\ approach using Data Envelopment Analysis (DEA)
}

\author{
Paulo Henrique da Silva ${ }^{\text {, }}$ Paulo Sérgio de Arruda Ignácio"I, \\ Alessandro Lucas da Silva ${ }^{\text {III }}$, Antônio Carlos Pacagnella Júnior ${ }^{\mathrm{IV}}$
}

\begin{abstract}
Resumo
O presente artigo refere-se à utilização da metodologia de análise de envoltória de dados (DEA) para determinar e comparar a eficiência técnica de clones de eucalipto dos gêneros Eucaliptus urophylla e Eucaliptus grandis, em escala comercial, produzidos no período de 2016 e 2017 e com idades de corte entre 5 e 7 anos. O plantio desses clones ocorreu em locais com solos arenosos, clima quente e temperado, destinados à produção de celulose e papel, pertencentes a uma empresa situada no interior do estado de São Paulo. Este estudo visa identificar quais clones apresentam ou não melhorias de eficiência técnica relacionadas a sua idade de corte, podendo auxiliar na tomada de decisão das melhores idades de corte e também quais clones deverão ser excluídos ou mantidos nos próximos ciclos produtivos. O cálculo da eficiência técnica e de seus valores atuais, folgas, alvos e benchmarks ao longo dos anos são determinados através das variáveis custo total; volume individual e volume produzido, sendo utilizado o modelo DEA BCC-O, em função da existência de pouca proporcionalidade de alguns pares input-output. Os clones CL02, CL03, CL05 e CL08 mostraram-se eficientes em ambas as idades de corte, caracterizando-os como possíveis benchmarks para os clones ineficientes. Já o clone CL04 foi o único cuja eliminação da lista de clones a serem utilizados nos próximos ciclos de plantio foi sugerida, apresentando diminuição de seu volume individual e densidade básica, quando ocorre o aumento de sua idade de corte. Conclui-se que a metodologia DEA é uma opção para o auxílio da tomada de decisão de quais clones de eucalipto devem ser utilizados, reduzidos e/ou eliminados de seu próximo ciclo, determinando quais são mais eficientes, verificando sua evolução em relação a sua idade de corte.
\end{abstract}

Palavras-chave: Benchmarks; Idade de corte; Tomada de decisão

\footnotetext{
Engenheiro de Produção, Me., Membro do grupo de pesquisa CENPRO - Centro de Pesquisa em Engenhraria de Produção da Faculdade de Ciências Aplicadas (FCA), Universidade Estadual de Campinas - UNICAMP, Rua Pedro Zaccaria, 1300, Campus Limeira, CEP 13484-350, Limeira (SP), Brasil. phs81@msn.com (ORCID: 0000-0002-3341-9030)

II Engenheiro de Produção Mecânica, Dr., Professor Assistente na Faculdade de Ciências Aplicadas (FCA), Universidade Estadual de Campinas UNICAMP, Rua Pedro Zaccaria, 1300, Campus Limeira, CEP 13484-350, Limeira (SP), Brasil. paulo.ignacio@fca.unicamp.br (ORCID: 0000-00034688-8693)

III Engenheiro de Produção Mecânica, Dr. Professor Assistente na Faculdade de Ciências Aplicadas (FCA), Universidade Estadual de Campinas UNICAMP, Rua Pedro Zaccaria, 1300, Campus Limeira, CEP 13484-350, Limeira (SP), Brasil. alessandro.silva@fca.unicamp.br (ORCID: 0000-00018282-8618)

IV Engenheiro Mecânico, Dr., Professor Assistente na Faculdade de Ciências Aplicadas (FCA), Universidade Estadual de Campinas - UNICAMP, Rua Pedro Zaccaria, 1300, Campus Limeira, CEP 13484-350, Limeira (SP), Brasil. antonio.junior@fca.unicamp.br (ORCID: 0000-0003-4485-2777)
} 


\begin{abstract}
This study aims to identify which clones present technical efficiency improvements regarding their cutting age, helping the decision-making as to their best cutting ages and which clones shall be either excluded or maintained in the next production cycles. The data envelopment analysis (DEA) methodology is discussed to determine and to compare the technical efficiency of eucalyptus clones of the genuses Eucaplyptus urophylla and Eucalyptus grandis in commercial scale. They were produced between 2016 and 2017, with cutting ages equal to five and seven years. Their plantation occurred in areas of sandy soils, and warm, temperate climate in order to produce cellulose and paper. The plantation belongs to a company located in the state of São Paulo. The calculation of the technical efficiency and its current values, clearances, targets and benchmarks throughout the years are determined by the variables total cost, individual volume, and produced volume. The DEA BCC-O model was employed due to the little proportionality of some input-output pairs. Clones CL02, CL03, CL05, and CL08 seemed to be efficient in both cutting ages. This indicated that they were likely benchmarks for the inefficient clones. However, clone CL04 was the only one which exclusion of the clone list to be used in the next plantation cycles was suggested since it presented a reduction in its individual volume and basic density, when an improvement in its cutting age occurs. We concluded that the DEA methodology is a good choice for helping the decisionmaking regarding which eucalyptus clones shall be used, reduced, and/or eliminated from the next cycle, determining which ones are the most efficient ones, verifying their evolution about their cutting ages.
\end{abstract}

Keywords: Benchmarks; Cutting age; Decision-making

\title{
Introdução
}

Com mais de 700 espécies originárias principalmente do continente australiano e com área total de plantação de mais de 19 milhões de hectares, o eucalipto é considerado uma das plantações mais comuns do mundo (YANG et al., 2017).

A introdução do eucalipto no Brasil deu-se a partir de 1904 por Edmundo Navarro de Andrade, por meio de reflorestamentos experimentais para a produção de dormentes e lenhas para a Companhia Paulista de Estradas de Ferro (MARTINI, 2004). No Brasil, os plantios de eucalipto correspondem a 5,7 milhões de hectares, estando situados especialmente nos estados de Minas Gerais (24\%), São Paulo (17\%) e no Mato Grosso do Sul (15\%) (INDÚSTRIA BRASILEIRA DE ÁRVORES, 2017).

Em virtude das favoráveis condições edafoclimáticas e também em função dos avanços tecnológicos na área de silvicultura, o gênero Eucalyptus tem se destacado no setor florestal brasileiro (SILVA, 2011). Com média de $35,7 \mathrm{~m}^{3} / \mathrm{ha} /$ ano, o Brasil liderou no ano de 2016 o ranking global de produtividade florestal para os plantios de eucalipto, sendo que nos últimos cinco anos sua produtividade aumentou em $0,2 \%$ ao ano (INDÚSTRIA BRASILEIRA DE ÁRVORES, 2017).

O gênero Eucalyptus é considerado uma opção de matéria-prima para a fabricação de celulose e papel, siderurgia, óleos essenciais, compensados, serrarias, mourões, entre outros fins (FERREIRA et al., 2014), buscando cada vez mais maiores eficiências em seu processo produtivo (GRATTAPAGLIA, 2014).

Dessa maneira, podemos verificar algumas ferramentas para determinar e avaliar a evolução ao longo do tempo da eficiência técnica de clones de eucalipto em escala comercial. Dentre essas ferramentas, podemos citar o método não paramétrico designado como DEA - Data Envelopment Analysis.

Também conhecida como análise de envoltória de dados, a DEA é uma metodologia não paramétrica aplicada a um conjunto de unidades tomadoras de decisões (DMUs) para a avaliação de suas fronteiras de produção, de forma a analisar sua eficiência relativa (SANTOS, 2011). 


\section{Revisão de literatura e objetivos}

O conceito de eficiência pode ser definido como a ótima combinação entre os insumos (inputs), para gerar o máximo de produto (outputs), de forma a minimizar a relação entre insumos e produtos (PEÑA, 2008).

As verificações do desempenho de unidades de produção são especialmente realizadas através de análise de fronteira, sendo as principais abordagens a paramétrica e a não-paramétrica. $\mathrm{Na}$ abordagem paramétrica, sua fronteira baseia-se em medidas de tendência central, enquanto a não paramétrica é baseada em medidas de valores externos observados (ARAÚJO; CARMONA, 2002).

Dessa forma, a análise de envoltória de dados (DEA) é um dos métodos relacionados aos estudos sobre a medição de eficiência em empresas (TITKO; STANKEVIČIENĖ; LĀCE, 2014). Por se tratar de um método não-paramétrico, não utiliza inferências estatísticas ou se apega a medidas de tendencial central, análise de regressões ou testes de coeficientes (FERREIRA; GOMES, 2009).

A DEA objetiva calcular a eficiência comparada entre unidades de produção (DMUs Decision Making Units), sendo uma metodologia inteiramente objetiva, dispensando a opinião do decisor (SENRA et al., 2007). Seus modelos clássicos são o CCR ou CRS (Constant Return to Scale) (CHARNES; COOPER; RHODES, 1978) e o BCC ou VRS (Variable Return to Scale) (BANKER; CHARNES; COOPER, 1984).

O modelo CCR faz uso de retornos de escala constantes, em que variações nas entradas (inputs) acarretam variações proporcionais nas saídas (outputs). A formulação do modelo DEACCR (MELLO; GOMES, 2004; VILELA; NAGANO; MERLO, 2007) é representada nas Equações 1, 2 e $3:$

$$
\max h_{0}=\sum_{j=1}^{s} u_{j} y_{j 0}
$$

Sujeito a:

$$
\begin{aligned}
& \sum_{i=1}^{m} v_{i} x_{i k}=1 \\
& \sum_{j=1}^{s} u_{j} y_{j k}-\sum_{i=1}^{m} v_{i} x_{i k} \leq 0 \\
& k=1, \ldots, n \\
& u_{j}, v_{i} \geq 0, \forall x, y
\end{aligned}
$$


Entretanto, o modelo BCC não apresenta proporcionalidade entre as entradas e as saídas (CORREIA; MELLO; MEZA, 2011). A formulação do modelo DEA-BCC (MELLO; GOMES, 2004; VILELA; NAGANO; MERLO, 2007) é retratada nas Equações 4, 5 e 6:

$$
\max h_{0}=\sum_{j=1}^{s} u_{j} y_{j 0}+u^{*}
$$

Sujeito a:

$$
\begin{aligned}
& \sum_{i=1}^{m} v_{i} x_{i 0}=1 \\
& \sum_{j=1}^{s} u_{j} y_{j k}-\sum_{i=1}^{n} v_{i} x_{i k}-u^{*} \leq 0 \\
& k=1, \ldots, n \\
& u_{j}, v_{i} \geq 0, \forall x, y \\
& u^{*} \in \Re
\end{aligned}
$$

Sendo:

$h_{0}$ eficiência da $\mathrm{DMU}_{0}$ em análise;

$x_{i k}$ representa o input ${ }_{i}$ da $\mathrm{DMU}_{\mathrm{k}}$;

$y_{j k}$ representa o output $t_{j}$ da $\mathrm{DMU}_{\mathrm{k}}$

$v_{i}$ representa o peso atribuído ao input;

$u_{j}$ representa o peso atribuído ao output $t_{j}$;

$u^{*}$ representa o fator de escala.

Os modelos DEA podem ser orientados em relação aos seus insumos (inputs) e produtos (outputs). Se o modelo for orientação ao input, seu objetivo será minimizar o input, produzindo a mesma quantidade de outputs. Quando o modelo for orientado ao output, seu objetivo será produzir o maior número de outputs, mantendo-se a quantidade de inputs (BARROS et al., 2010).

Também é possível identificar, por esses modelos, as unidades de referência, ou benchmarks para as unidades produtivas consideradas ineficientes, ou seja, promover um comparativo para essas organizações com a finalidade de melhorar sua eficiência (SILVEIRA; MEZA; MELLO, 2012).

Assim, objetivo principal deste estudo consiste em determinar e comparar a eficiência técnica de clones de eucalipto em escala comercial produzidos em solo arenoso no período de 2016 e 2017, com idades de corte que variam entre 5 e 7 anos. Essas análises objetivam identificar quais clones de eucalipto apresentam ou não melhorias em sua eficiência técnica em função de sua idade de corte, podendo auxiliar na tomada de decisão de quais seriam as melhores idades de corte para os clones estudados e também se esses deverão ser excluídos ou mantidos nos próximos ciclos produtivos. 


\section{Materiais e métodos}

\section{Metodologia}

Este estudo utiliza dados obtidos no banco de dados de uma empresa florestal que produz eucalipto dos gêneros Eucaliptus urophylla e Eucaliptus grandis para sua planta de produção de celulose e papel, localizada no interior do estado de São Paulo, com clima quente e temperado, sendo analisados e avaliados para o tipo de solo com características arenosas e com volume médio de em torno de $35 \mathrm{~m}^{3} /$ ha/ano. Seu escopo propõe a determinação e a comparação da eficiência técnica de clones de eucalipto em escala comercial.

\section{Levantamento das informações}

O levantamento das informações ocorre por meio da base de dados da empresa objeto estudo para os anos de 2016 e 2017, sendo essas informações analisadas e agrupadas, conforme apresentado a seguir:

- Por ano de recebimento, sendo utilizados os anos de 2016 e 2017;

- Por tipo de clone de eucalipto, dos gêneros Eucaliptus urophylla e Eucaliptus grandis;

- Utilizando somente madeiras localizadas no Estado de São Paulo;

- Por Horto e Talhões (Local de plantio);

- Com idade de corte entre 5 e 7 anos;

- Somente florestas de parcerias e próprias da empresa objeto de estudo;

- Somente áreas com solos de característica arenosa, conforme classificação da empresa estudada, totalizando uma área de 8.124,80 ha.

Esses dados são compostos pelas seguintes variáveis: custo total (R\$); densidade básica $\left(\mathrm{kg} / \mathrm{m}^{3}\right)$; idade de corte (anos); volume individual $\left(\mathrm{dm}^{3}\right)$ e volume produzido $\left(\mathrm{m}^{3}\right)$.

O volume produzido em metros cúbicos $\left(\mathrm{m}^{3}\right)$ são valores obtidos pós colheita, e coletados através do sistema de medição de volume Logmeter da empresa.

A variável volume individual, comumente utilizada em metro cúbico $\left(\mathrm{m}^{3}\right)$, foi convertida e utilizada em decímetro cúbico $\left(\mathrm{dm}^{3}\right)$. Essa conversão foi necessária, para se ter uma maior precisão e sensibilidade durante a aplicação dos modelos DEA, em função dos valores encontrados na base de dados serem muito próximos entre si, podendo inviabilizar a utilização dessa variável tão importante.

A variável custo total ( $\mathrm{R} \$$ ) foi substituída por custos similares a esse processo e disponível na literatura acadêmica (WILCKEN et al., 2008), visto que essa variável possui caráter sigiloso, sendo esses valores calculados usando o conceito de valor futuro (VF) e corrigidos para o ano de 2016. Esse ajuste ocorrido para os anos avaliados visam à padronização dos valores ao longo desses anos, sendo atualizados através do índice geral de preços - disponibilidade interna (IGPDI), conforme apresentado na Equação 7.

$$
M=P .(1+i)^{n}
$$

Onde:

$M$ representa o montante ao final da aplicação;

$P$ representa o principal, o valor inicial em Janeiro de 2008 que corresponde à $R \$ 2.514,00$ (WILCKEN et al., 2008);

$i$ representa a taxa de juros;

$n$ representa o prazo. 
Na sequência é realizado o cálculo dos custos totais para cada área plantada, multiplicandose o valor de $\mathrm{R} \$ 4.437,30$ por hectare $(\mathrm{R} \$ / \mathrm{ha})$, definido através da Equação 7 , pela área plantada em hectares (ha) em função do tipo de clone e idade de corte, conforme exibido na Equação 8.

$$
\text { Custo total }(\mathrm{R} \$)=\text { Custo corrigido } 2016\left(\frac{R \$}{H a}\right) \times \text { Área plantada (Ha) }
$$

Em seguida, foi realizada análise das variáveis para verificação da existência de valores faltantes na base de dados, sendo esse período excluído da base caso ocorra falta de algum dado.

\section{Análise das variáveis}

Para as variáveis utilizadas, foi realizada a comparação das entradas e das saídas, individualmente, para idades entre 5 e 7 anos, por meio da ferramenta gráfico de dispersão por tipo de solo. Na sequência foi realizada a análise de correlação das entradas e das saídas, individualmente, para cada par input-output, verificando se teve ocorrência de proporcionalidade entre essas variáveis.

Através dessas análises é possível determinar a existência de proporcionalidade entre os pares inputs-outputs para ambas as idades, de forma a identificar seus rendimentos de escala e posteriormente sua orientação com input ou output (MELLO et al., 2006).

Em seguida deve-se definir a quantidade de DMUs a ser utilizada, sendo que a essas deverão ser de pelo menos três vezes o número de entradas e saídas (DYSON et al., 2001), e as variáveis avaliadas devem ser selecionadas de acordo com a sua finalidade de utilização, de maneira a classificá-las como: entrada, as variáveis que visam à minimização, e variáveis de saídas, as que visam à maximização (GOMES; MANGABEIRA, 2004).

Na sequência, de maneira a auxiliar para a definição e determinar quais variáveis devem ser utilizadas no modelo, é realizada a análise de correlação entre as variáveis, para ambas as idades. Essa análise pretende, através da criação de uma matriz de inter correlação entre essas variáveis, de forma a determinar o grau de correlação entre as duas variáveis analisadas, distinguir o desempenho de cada DMU, classificando as variáveis como redundantes ou irrelevantes.

Sua classificação é efetivada pelo meio da verificação do coeficiente de correlação de Spearman com o auxílio do software Minitab licenciado, sendo que algumas variáveis apresentam seus dados como não normais $(\mathrm{p}<0,05)$. Para as variáveis que apresentarem correlação maior ou igual a 0,75 ou que apresentaram uma correlação negativa entre input-output, essas serão retiradas do modelo utilizado.

Finalizando, para efetuar os cálculos dos modelos será utilizado o software livre SIAD Sistema Integrado de Apoio a Decisão (MEZA et al., 2005), para cada um dos modelos, de forma a determinar as DMUs classificadas como eficientes e ineficientes, gerando seus pesos e também seus benchmarks.

\section{Resultados e discussões}

Através das análises das variáveis foi possível determinar a existência de pouca proporcionalidade de alguns pares input-output para ambas a idades avaliadas, caracterizando rendimentos de escalas variáveis, sendo o melhor modelo a ser utilizado o DEA-BCC, com orientação ao output. 
As DMUs utilizadas apresentaram mesma utilização, variando somente em sua extensão de plantio e em quantidade de mudas plantadas, sendo representadas pelos clones de eucalipto utilizados pela empresa no período avaliado. Os clones de eucaliptos utilizados nos modelos foram CL01, CL02, CL03, CL04, CL05, CL06, CL07, CL08 e CL09. Para os modelos utilizados define-se que a quantidade de DMUs deverá ser de pelo menos três vezes o número de entradas e saídas, e as variáveis avaliadas devem ser selecionadas de acordo com a sua finalidade de utilização, sendo apresentadas no Tabela 1.

Tabela 1 - Classificação das variáveis como Input-Output para utilização nos modelos DEA

Table 1 - Classification of variables as Input-Output for use in DEA models

\begin{tabular}{cc}
\hline Tipo & Variáveis \\
\hline \multirow{2}{*}{ Input } & $\begin{array}{c}\text { Custo total }(\mathrm{R} \$) \\
\text { Idade de corte }(\mathrm{Anos})\end{array}$ \\
\hline \multirow{2}{*}{ Output } & Volume individual $\left(\mathrm{dm}^{3}\right)$ \\
& Densidade Básica $\left(\mathrm{Kg} / \mathrm{m}^{3}\right)$ \\
& Volume produzido $\left(\mathrm{m}^{3}\right)$ \\
\hline
\end{tabular}

Fonte: Autores (2018)

Os dados foram agrupados de acordo com o tipo de modelo DEA BCC-O em função de sua idade de corte, sendo utilizado para as variáveis custo total (R\$) e volume produzido $\left(\mathrm{m}^{3}\right)$ seus valores acumulados, e para as variáveis idade de corte (Anos), volume individual $\left(\mathrm{dm}^{3}\right)$ e densidade básica $\left(\mathrm{Kg} / \mathrm{m}^{3}\right)$ a média de seus dados, conforme apresentado na Tabela 2.

Tabela 2 - Classificação das possíveis variáveis a serem utilizadas nos modelos DEA

Table 2 - Classification of possible variables to be used in DEA models

\begin{tabular}{lccccc}
\hline \multicolumn{5}{c}{ Idade de corte entre 5 à 6 anos } \\
\hline DMUs & $\begin{array}{c}\text { Custo total } \\
(\mathbf{R} \mathbf{\$} \times \mathbf{~ 1 . 0 0 0})\end{array}$ & $\begin{array}{c}\text { Idade } \\
(\text { Anos })\end{array}$ & $\begin{array}{c}\text { Volume } \\
\text { Individual } \\
\left(\mathbf{d m}^{\mathbf{3}}\right)\end{array}$ & $\begin{array}{c}\text { Densidade } \\
\text { básica } \\
\left(\mathbf{K g} / \mathbf{m}^{\mathbf{3}}\right)\end{array}$ & $\begin{array}{c}\text { Volume } \\
\text { produzido } \\
\left(\mathbf{m}^{\mathbf{3}}\right)\end{array}$ \\
\hline CL01 & $2.327,49$ & 5,57 & 166,84 & 481,47 & $108.700,39$ \\
CL02 & 681,70 & 5,56 & 157,35 & 483,21 & $34.251,11$ \\
CL03 & $2.113,44$ & 5,62 & 212,40 & 472,36 & $115.760,47$ \\
CL04 & $1.147,04$ & 5,44 & 163,76 & 504,26 & $53.135,92$ \\
CL05 & 40,25 & 5,08 & 111,56 & 472,82 & $1.455,54$ \\
CL06 & $3.152,03$ & 5,48 & 145,49 & 494,36 & $130.937,12$ \\
CL07 & 358,44 & 5,75 & 120,03 & 467,5 & $15.388,34$ \\
CL08 & $11.234,17$ & 5,48 & 155,77 & 485,06 & $488.808,63$ \\
CL09 & $4.022,85$ & 5,48 & 150,56 & 488,33 & $171.464,32$
\end{tabular}


Tabela 2 - Conclusão ...

Table 2 - Conclusion ...

\begin{tabular}{lccccc}
\hline \multicolumn{5}{c}{ Idade de corte entre 6 à 7 anos } \\
\hline DMUs & $\begin{array}{c}\text { Custo total } \\
(\mathbf{R} \mathbf{\$} \mathbf{1} \text { 1.000 })\end{array}$ & $\begin{array}{c}\text { Idade } \\
(\text { Anos })\end{array}$ & $\begin{array}{c}\text { Volume } \\
\text { Individual } \\
\left(\mathbf{d m}^{\mathbf{3}}\right)\end{array}$ & $\begin{array}{c}\text { Densidade } \\
\text { básica } \\
\left(\mathbf{K g} / \mathbf{m}^{\mathbf{3}}\right)\end{array}$ & $\begin{array}{c}\text { Volume } \\
\text { produzido } \\
\left(\mathbf{m}^{\mathbf{3}}\right)\end{array}$ \\
\hline CL01 & 628,19 & 6,23 & 164,17 & 485,99 & $32.046,52$ \\
CL02 & 444,62 & 6,66 & 187,94 & 488,95 & $23.278,30$ \\
CL03 & $1.428,01$ & 6,49 & 215,17 & 485,18 & $86.438,59$ \\
CL04 & $1.144,07$ & 6,15 & 152,31 & 503,89 & $48.540,91$ \\
CL05 & 293,31 & 6,39 & 162,29 & 497,25 & $15.143,15$ \\
CL06 & 948,25 & 6,28 & 205,06 & 504,04 & $55.353,52$ \\
CL07 & $1.586,56$ & 6,35 & 158,38 & 488,88 & $67.553,00$ \\
CL08 & $3.463,22$ & 6,16 & 159,68 & 489,17 & $154.953,56$ \\
CL09 & $1.038,50$ & 6,15 & 164,99 & 494,8 & $45.227,83$ \\
\hline
\end{tabular}

Fonte: Autores (2018)

Empregando-se os dados apresentados na Tabela 2, foi realizada a análise de correlação entre as variáveis, para ambas as idades. Sua classificação foi definida através da verificação do coeficiente de correlação de Spearman. A Tabela 3 apresenta os valores de correlação para ambas as idades:

Tabela 3 - Análise das correlações entre varáveis para ambas as idades

Table 3 - Analysis of the correlations among variables for both ages

\begin{tabular}{|c|c|c|c|c|c|}
\hline \multicolumn{6}{|c|}{ Idade de corte entre 5 à 6 anos } \\
\hline $\begin{array}{l}\text { Correlação } \\
\text { Spearman }\end{array}$ & $\begin{array}{l}\text { Custo total } \\
(\mathbf{R} \$ \times 1.000)\end{array}$ & $\begin{array}{l}\text { Idade } \\
\text { (anos) }\end{array}$ & $\begin{array}{c}\text { Volume } \\
\text { Individual } \\
\left(\mathbf{d m}^{3}\right) \\
\end{array}$ & $\begin{array}{c}\text { Densidade } \\
\text { básica } \\
\left(\mathrm{Kg} / \mathbf{m}^{3}\right)\end{array}$ & $\begin{array}{c}\text { Volume } \\
\text { produzido } \\
\left(\mathbf{m}^{3}\right)\end{array}$ \\
\hline $\begin{array}{l}\text { Custo total } \\
(\mathbf{R} \$ \times 1.000)\end{array}$ & 1,000 & & & & \\
\hline Idade (anos) & $-0,034$ & 1,000 & & & \\
\hline $\begin{array}{l}\text { Volume } \\
\text { Individual } \\
\left(\mathbf{d m}^{3}\right)\end{array}$ & 0,283 & 0,339 & 1,000 & & \\
\hline $\begin{array}{l}\text { Densidade } \\
\text { básica } \\
\left(\mathbf{K g} / \mathbf{m}^{3}\right)\end{array}$ & 0,517 & $-0,627$ & 0,083 & 1,000 & \\
\hline $\begin{array}{l}\text { Volume } \\
\text { produzido } \\
\left(\mathbf{m}^{\mathbf{3}}\right)\end{array}$ & 0,983 & $-0,017$ & 0,300 & 0,483 & 1,000 \\
\hline
\end{tabular}


Tabela 3 - Conclusão ...

Table 3 - Conclusion ...

\begin{tabular}{|c|c|c|c|c|c|}
\hline \multicolumn{6}{|c|}{ Idade de corte entre 6 à 7 anos } \\
\hline $\begin{array}{l}\text { Correlação } \\
\text { Spearman }\end{array}$ & $\begin{array}{l}\text { Custo total } \\
(\mathbf{R} \$ \times 1.000)\end{array}$ & $\begin{array}{l}\text { Idade } \\
\text { (anos) }\end{array}$ & $\begin{array}{c}\text { Volume } \\
\text { Individual } \\
\left(\mathbf{d m}^{\mathbf{3}}\right)\end{array}$ & $\begin{array}{c}\text { Densidade } \\
\text { básica }(\mathrm{Kg} / \\
\left.\mathbf{m}^{3}\right)\end{array}$ & $\begin{array}{c}\text { Volume } \\
\text { produzido } \\
\left(\mathbf{m}^{\mathbf{3}}\right)\end{array}$ \\
\hline $\begin{array}{l}\text { Custo total } \\
(\mathbf{R} \$ \times 1.000)\end{array}$ & 1,000 & & & & \\
\hline Idade (anos) & $-0,343$ & 1,000 & & & \\
\hline $\begin{array}{l}\text { Volume } \\
\text { Individual } \\
\left(\mathbf{d m}^{\mathbf{3}}\right)\end{array}$ & $-0,300$ & 0,494 & 1,000 & & \\
\hline $\begin{array}{l}\text { Densidade } \\
\text { básica } \\
\left(\mathrm{Kg} / \mathbf{m}^{3}\right)\end{array}$ & $-0,233$ & $-0,418$ & $-0,217$ & 1,000 & \\
\hline $\begin{array}{l}\text { Volume } \\
\text { produzido } \\
\left(\mathbf{m}^{3}\right)\end{array}$ & 0,933 & $-0,192$ & $-0,033$ & $-0,200$ & 1,000 \\
\hline
\end{tabular}

Fonte: Autores (2018)

É possível verificar, pela Tabela 3, que as variáveis Densidade básica $\left(\mathrm{Kg} / \mathrm{m}^{3}\right)$ e Idade (anos) foram as únicas variáveis que apresentaram valores negativos para ambas as idades, de modo que essas foram excluídas do modelo DEA. A Tabela 4 apresenta as variáveis classificadas e utilizadas nos modelos DEA BCC-O.

Tabela 4 - Classificação das variáveis utilizadas nos modelos DEA através das análises de correlação

Table 4 - Classification of variables used in the DEA models through correlation analysis

\begin{tabular}{|c|c|c|c|c|c|c|c|}
\hline \multicolumn{4}{|c|}{ Idade de corte entre 5 à 6 anos } & \multicolumn{4}{|c|}{ Idade de corte entre 6 à 7 anos } \\
\hline DMUs & $\begin{array}{l}\text { Custo total } \\
(\mathbf{R} \$ \times 1.000)\end{array}$ & $\begin{array}{c}\text { Volume } \\
\text { Individual } \\
\left(\mathbf{d m}^{\mathbf{3}}\right)\end{array}$ & $\begin{array}{l}\text { Volume } \\
\text { produzido } \\
\left(\mathbf{m}^{3}\right)\end{array}$ & DMUs & $\begin{array}{l}\text { Custo total } \\
(\mathbf{R} \$ \times 1.000)\end{array}$ & $\begin{array}{c}\text { Volume } \\
\text { Individual } \\
\left(\mathbf{d m}^{\mathbf{3}}\right)\end{array}$ & $\begin{array}{c}\text { Volume } \\
\text { produzido } \\
\quad\left(\mathbf{m}^{\mathbf{3}}\right)\end{array}$ \\
\hline CL01 & $2.327,49$ & 166,84 & $108.700,39$ & CL01 & 628,19 & 164,17 & $32.046,52$ \\
\hline CLO2 & 681,70 & 157,35 & $34.251,11$ & CL02 & 444,62 & 187,94 & $23.278,30$ \\
\hline CL03 & $2.113,44$ & 212,40 & $115.760,47$ & CL03 & $1.428,01$ & 215,17 & $86.438,59$ \\
\hline CLO4 & $1.147,04$ & 163,76 & $53.135,92$ & CL04 & $1.144,07$ & 152,31 & $48.540,91$ \\
\hline CL05 & 40,25 & 111,56 & $1.455,54$ & CL05 & 293,31 & 162,29 & $15.143,15$ \\
\hline CLO6 & $3.152,03$ & 145,49 & $130.937,12$ & CL06 & 948,25 & 205,06 & $55.353,52$ \\
\hline CL07 & 358,44 & 120,03 & $15.388,34$ & CL07 & $1.586,56$ & 158,38 & $67.553,00$ \\
\hline CLO8 & $11.234,17$ & 155,77 & $488.808,63$ & CL08 & $3.463,22$ & 159,68 & $154.953,56$ \\
\hline CLO9 & $4.022,85$ & 150,56 & $171.464,32$ & CL09 & $1.038,50$ & 164,99 & $45.227,83$ \\
\hline
\end{tabular}

Fonte: Autores (2018) 
$\mathrm{Na}$ sequência, foram aplicados os modelos DEA BCC-O para determinar as medições das eficiências para ambas as idades avaliadas no Software SIAD, sendo obtidos os seguintes resultados apresentados na Figura 1.

Figura 1 - Comparativo das eficiências por idade e tipo de clone geradas pelos modelos DEA

Figure 1 - Comparison of efficiencies according to age and clone type generated by the DEA models

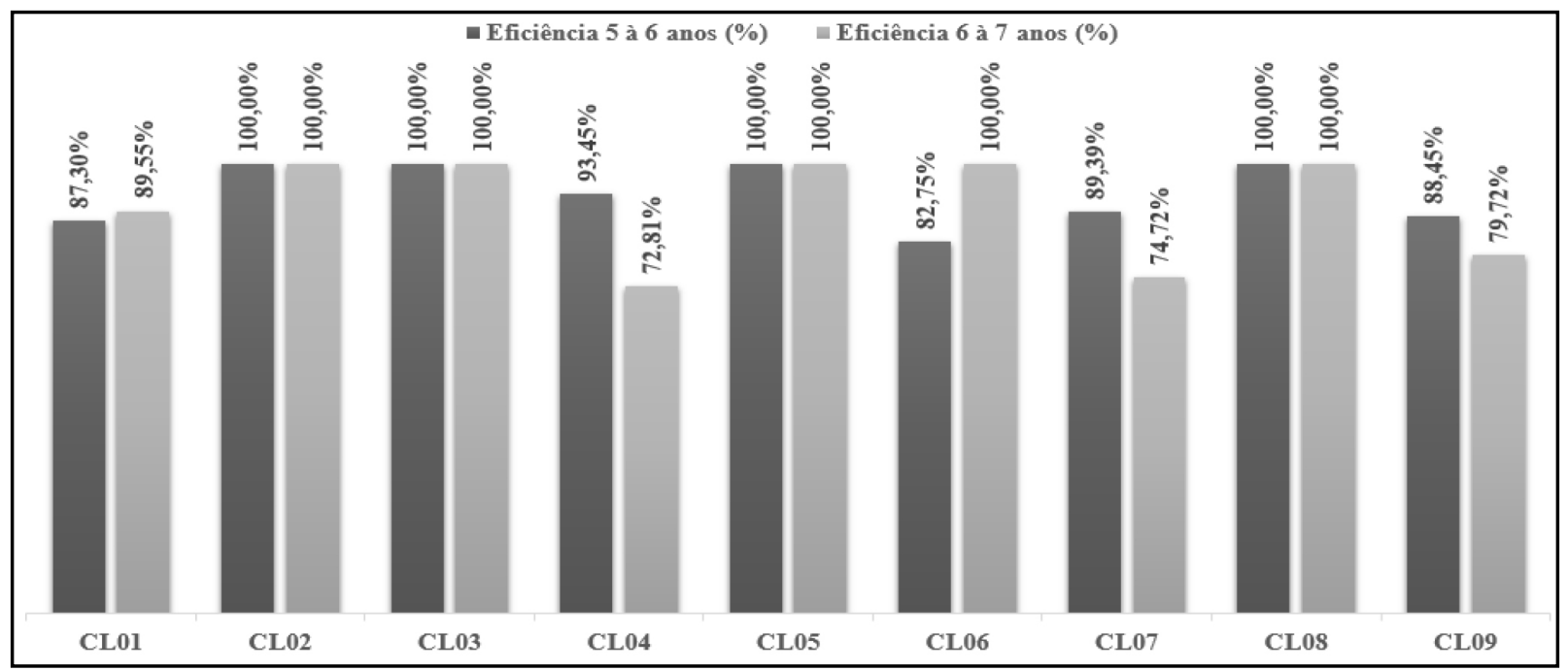

Fonte: Autores (2018)

Na Figura 1, verifica-se que, para os clones com idades de corte entre 5 e 6 anos, dos 9 clones avaliados, 4 deles se mostraram eficientes, ou seja, $44,45 \%$ dos clones. Quanto a sua variação, é possível verificar uma diferença entre a maior e menor eficiente entre $100,00 \%$ e $82,75 \%$, respectivamente.

Também é possível verificar que os clones CL02, CL03, CL05 e CL08 apresentam as melhores eficientes para os clones com idades de corte entre 5 e 6 anos. Já o clone CL06 é o clone que apresenta a menor eficiência entre os clones avaliados, com eficiência de $82,75 \%$.

Para os clones com idade de corte entre 6 e 7 anos, é possível verificar que, do total de 9 clones analisados, 5 deles se mostraram eficientes, o que corresponde a 55,56\%. A diferença entre os clones de maior e menor eficiente é de $100,00 \%$ e $72,81 \%$, sendo o clone CL04 o que apresenta a menor eficiência.

Constata-se também que os clones CL02, CL03, CL05, CL06 e CL08 são os que apresentam as melhores eficiências com idade de corte entre 6 e 7 anos.

Os clones CL02, CL03, CL05 e CL08 mostram-se eficientes em ambas as idades de corte, sendo caracterizados como possíveis benchmarks diante dos clones classificados como ineficientes. Suas propriedades podem ser analisadas em estudos futuros, podendo ser referência para o desenvolvimento de clones mais eficientes.

Entretanto, conforme apresentado na Tabela 2, a variação de idade entre esses clones é de 0,54 anos para as idades de corte entre 5 e 6 anos, sendo o clone CL05 o que apresenta a menor idade média de corte de 5,08 anos. Para as idades de corte entre 6 e 7 anos, a variação de idade entre esses clones é de 0,50 anos, sendo os clones CL04 e CL09 os que apresentam a menor idade média de corte de 6,15 anos. 
Com relação a seus volumes individuais, verifica-se que os clones CL02 e CL05 são os clones que apresentam os maiores aumentos conforme sua idade média de corte é aumentada, correspondente a $16,28 \%$ e a $31,26 \%$, respectivamente. Para a variável Densidade básica é possível verificar que os clones CL03 e CL05 são os que apresentam maior aumento, que corresponde a $2,64 \%$ e $4,91 \%$.

Já os clones CL01 e CL06 são os únicos clones que, conforme é aumentada sua idade de corte, também apresentam aumentos em suas eficiências. Dentre esses clones, o clone CL06 é o que apresenta maior evolução em suas eficiências, com aumento de $82,75 \%$ quando sua idade de corte está entre 5 e 6 anos e 100,00\% quando sua idade está entre 6 e 7 anos (Figura 1).

Para a variável Volume individual, é possível verificar que o clone CL06 apresenta um aumento de $29,05 \%$ conforme aumenta a idade média de corte. Entretanto, o clone CL01 apresenta uma diminuição de $-1,63 \%$ em seu volume individual. Em relação à variável Densidade básica, é possível verificar para ambos os clones aumentos em função do aumento da idade média de corte, apresentando o valor de 0,93\% para o clone CL01 e 1,92\% para o clone CL06.

Porém, os clones CL01, CL04, CL07 e CL09 são os que se apresentam ineficientes em ambas as idades de corte, sendo que os clones CL04, CL07 e CL09, ao aumentar de idade de corte, ocorre a diminuição de suas eficiências.

A variação de idade entre esses clones é de 0,31 anos para as idades de corte entre 5 e 6 anos, sendo o clone CL07 o que apresenta a maior idade média entre todos os clones avaliados com idade de 5,75 anos. Para as idades de corte entre 6 e 7 anos, os clones CL04 e CL09 são os que apresentam as menores idades entre os clones analisados, ambos com idades de 6,15 anos.

Para a variável Volume individual, é possível observar que os clones CL07 e CL09 exibem aumento conforme ocorrem aumentos em função do aumento da idade média de corte, apresentando os valores de $24,21 \%$ e $8,75 \%$, respectivamente. No entanto, o clone CL04 apresenta uma diminuição de $-7,52 \%$ em seu Volume individual. Com relação à variável Densidade básica, é verificado que o clone CL04 também apresenta uma diminuição em função do aumento da idade média de corte, com valor de $-0,07 \%$.

Dessa forma, o clone CL04 pode ser assinalado como o clone a ser possivelmente eliminado da lista de clones a serem utilizados nos próximos ciclos de plantio, visto que apresenta uma diminuição de suas variáveis Volume individual e Densidade básica conforme ocorre o aumento de sua idade de corte. Caso opte-se pela sua utilização, buscando a obtenção de uma maior eficiência e produtividade, deve-se optar pelo seu corte com idades entre 5 e 6 anos. Já para o clone CL01 deve ser escolhido pelo seu corte com idades entre 6 e 7 anos de idade e para os demais clones com idade entre 5 e 6 anos.

Com relação aos valores atuais, folgas e alvos gerados pelos modelos, a Tabela 5 representa de maneira detalhada esses resultados.

Pelos valores atuais, folgas e alvos obtidos pelo modelo DEA BCC-O e apresentados na Tabela 5, é possível observar que, para as análises com Idade de corte entre 5 e 6 anos, os clones CL06 e CL09 são os clones que apresentam os menores percentuais com relação à variável Volume individual, apresentando valores de $-41,56 \%$ e -33,20\%, respectivamente. Já os clones CL06 e CL07 apresentam para a variável Volume produzido os menores percentuais, apresentando valores de $-20,85 \%$ e $-15,18 \%$.

Analisando as idades entre 6 e 7 anos, é possível verificar que os clones CL04 e CL07 são os que apresentam os menores percentuais com relação à variável Volume individual, apresentando valores de $-37,34 \%$ e $-33,83 \%$. Para a variável Volume produzido, os clones CL04 e CL09 apresentam os menores percentuais com valores de $-40,17 \%$ e $-35,32 \%$, respectivamente. 
Tabela 5 - Valores atuais, folgas e alvos por tipo de clone e idades obtidos pelos modelos DEA

Table 5 - Current values, clearances, and targets by clone type and age obtained by the DEA models

\begin{tabular}{|c|c|c|c|c|c|c|c|c|c|c|c|}
\hline \multicolumn{6}{|c|}{ Alvos 5 à 6 anos } & \multicolumn{6}{|c|}{ Alvos 6 à 7 anos } \\
\hline DMU & Variável & Atual & Folga & Alvo & $\begin{array}{c}\text { Diferença } \\
(\%)\end{array}$ & DMU & Variável & Atual & Folga & Alvo & $\begin{array}{c}\text { Diferença } \\
(\%)\end{array}$ \\
\hline \multirow{3}{*}{ CL01 } & $\mathrm{CT}$ & $2.327,49$ & 0,00 & $2.327,49$ & 0,00 & & $\mathrm{CT}$ & 628,19 & 0,00 & 628,19 & 0,00 \\
\hline & VInd & 166,84 & 19,96 & 211,07 & $-26,51$ & CL01 & VInd & 164,17 & 0,00 & 183,32 & $-11,66$ \\
\hline & VP & $108.700,39$ & 0,00 & $124.515,57$ & $-14,55$ & & VP & $32.046,52$ & 0,00 & $35.784,39$ & $-11,66$ \\
\hline \multirow{3}{*}{ CLO2 } & $\mathrm{CT}$ & 681,70 & 0,00 & 681,70 & 0,00 & & $\mathrm{CT}$ & 444,62 & 0,00 & 444,62 & 0,00 \\
\hline & VInd & 157,35 & 0,00 & 157,35 & 0,00 & CLO2 & VInd & 187,94 & 0,00 & 187,94 & 0,00 \\
\hline & VP & $34.251,11$ & 0,00 & $34.251,11$ & 0,00 & & VP & $23.278,30$ & 0,00 & $23.278,30$ & 0,00 \\
\hline \multirow{3}{*}{ CL03 } & $\mathrm{CT}$ & $2.113,44$ & 0,00 & $2.113,44$ & 0,00 & & $\mathrm{CT}$ & $1.428,01$ & 0,00 & $1.428,01$ & 0,00 \\
\hline & VInd & 212,40 & 0,00 & 212,40 & 0,00 & CL03 & VInd & 215,17 & 0,00 & 215,17 & 0,00 \\
\hline & VP & $115.760,47$ & 0,00 & $115.760,47$ & 0,00 & & VP & $86.438,59$ & 0,00 & $86.438,59$ & 0,00 \\
\hline \multirow{3}{*}{ CLO4 } & $\mathrm{CT}$ & $1.147,04$ & 0,00 & $1.147,04$ & 0,00 & & $\mathrm{CT}$ & $1.144,07$ & 0,00 & $1.144,07$ & 0,00 \\
\hline & VInd & 163,76 & 0,00 & 175,24 & $-7,01$ & CLO4 & VInd & 152,31 & 0,00 & 209,19 & $-37,34$ \\
\hline & VP & $53.135,92$ & 3881,45 & $60.743,04$ & $-14,32$ & & VP & $48.540,91$ & 1373,79 & $68.041,13$ & $-40,17$ \\
\hline \multirow{3}{*}{ CL05 } & $\mathrm{CT}$ & 40,25 & 0,00 & 40,25 & 0,00 & & $\mathrm{CT}$ & 293,31 & 0,00 & 293,31 & 0,00 \\
\hline & VInd & 111,56 & 0,00 & 111,56 & 0,00 & CL05 & VInd & 162,29 & 0,00 & 162,29 & 0,00 \\
\hline & VP & $1.455,54$ & 0,00 & $1.455,54$ & 0,00 & & VP & $15.143,15$ & 0,00 & $15.143,15$ & 0,00 \\
\hline \multirow{3}{*}{ CLO6 } & $\mathrm{CT}$ & $3.152,03$ & 0,00 & $3.152,03$ & 0,00 & & $\mathrm{CT}$ & 948,25 & 0,00 & 948,25 & 0,00 \\
\hline & VInd & 145,49 & 30,12 & 205,95 & $-41,56$ & CL06 & VInd & 205,06 & 0,00 & 205,06 & 0,00 \\
\hline & VP & $130.937,12$ & 0,00 & $158.240,12$ & $-20,85$ & & VP & $55.353,52$ & 0,00 & $55.353,52$ & 0,00 \\
\hline \multirow{3}{*}{ CL07 } & $\mathrm{CT}$ & 358,44 & 0,00 & 358,44 & 0,00 & & $\mathrm{CT}$ & $1.586,56$ & 40719,87 & $1.586,56$ & 2,57 \\
\hline & VInd & 120,03 & 0,00 & 134,27 & $-11,87$ & CL07 & VInd & 158,38 & 0,00 & 211,96 & $-33,83$ \\
\hline & VP & $15.388,34$ & 509,47 & $17.724,01$ & $-15,18$ & & VP & $67.553,00$ & 0,00 & $90.405,14$ & $-33,83$ \\
\hline \multirow{3}{*}{ CL08 } & $\mathrm{CT}$ & $11.234,17$ & 0,00 & $11.234,17$ & 0,00 & & $\mathrm{CT}$ & $3.463,22$ & 0,00 & $3.463,22$ & 0,00 \\
\hline & VInd & 155,77 & 0,00 & 155,77 & 0,00 & CL08 & VInd & 159,68 & 0,00 & 159,68 & 0,00 \\
\hline & VP & $488.808,63$ & 0,00 & $488.808,63$ & 0,00 & & VP & $154.953,56$ & 0,00 & $154.953,56$ & 0,00 \\
\hline \multirow{3}{*}{ CL09 } & $\mathrm{CT}$ & $4.022,85$ & 0,00 & $4.022,85$ & 0,00 & & $\mathrm{CT}$ & $1.038,50$ & 0,00 & $1.038,50$ & 0,00 \\
\hline & VInd & 150,56 & 30,32 & 200,54 & $-33,20$ & CL09 & VInd & 164,99 & 0,00 & 206,96 & $-25,44$ \\
\hline & VP & $171.464,32$ & 0,00 & $193.857,62$ & $-13,06$ & & VP & $45.227,83$ & 4468,00 & $61.201,38$ & $-35,32$ \\
\hline
\end{tabular}

Fonte: Autores (2018)

Em que CT = Custo total $(\mathrm{R} \$ \mathrm{x} 1.000) ; \mathrm{VInd}=$ Volume individual $\left(\mathrm{dm}^{3}\right) ; \mathrm{VP}=$ Volume produzido $\left(\mathrm{m}^{3}\right)$. 
Dessa forma, para os clones classificados como ineficientes existe a possibilidade de acréscimo em seus Volumes individuais e Volumes produzidos, buscando, assim, adentrar no grupo dos clones eficientes. Para esses clones, classificados como ineficientes, seus benchmarks e seus índices de referência são apresentados na Tabela 6. Tabela 6 - Benchmarks por tipo de clone e idades para os clones classificados como
ineficientes pelos modelos DEA

Table 6 - Benchmarks according to clone type and age for clones classified as inefficient by the DEA models

\begin{tabular}{ccccc|cccccc}
\hline \multicolumn{5}{c|}{ Benchmarks 5 à $\mathbf{6}$ anos (\%) } & \multicolumn{6}{c}{ Benchmarks 6 à 7 anos (\%) } \\
\hline DMU & CL02 & CL03 & CL05 & CL08 & DMU & CL02 & CL03 & CL05 & CL06 & CL08 \\
\hline CL01 & 0,00 & 97,65 & 0,00 & 2,35 & CL01 & 29,15 & 25,63 & 45,22 & 0,00 & 0,00 \\
CL04 & 67,50 & 32,50 & 0,00 & 0,00 & CL04 & 0,00 & 40,82 & 0,00 & 59,18 & 0,00 \\
CL06 & 0,00 & 88,61 & 0,00 & 11,39 & CL07 & 0,00 & 94,21 & 0,00 & 0,00 & 5,79 \\
CL07 & 49,61 & 0,00 & 50,39 & 0,00 & CL09 & 0,00 & 18,81 & 0,00 & 81,19 & 0,00 \\
CL09 & 0,00 & 79,07 & 0,00 & 20,93 & & & & & & \\
\hline
\end{tabular}

Fonte: Autores (2018)

Os clones benchmarks, apresentados na Tabela 6, são considerados eficientes e são utilizados como referência para o grupo de clones classificados como ineficientes. Verifica-se que, paraos clones com Idade de corte entre 5 e 6 anos, o clone CL03 é o principal benchmark, sendo referência para os clones CL01, CL06 e CL09, apresentando valores de 97,65\%, 88,61\% e 79,07\%. Já os clones CL02 e CL05 são referências para os clones CL04 e CL05, apresentando valores $67,50 \%$ e $50,39 \%$, respectivamente.

Para os clones com Idade de corte entre 6 e 7 anos, é possível verificar que o clone CL06 é considerado como principal benchmark para os clones CL04 (59,18\%) e CL09 (81,19\%). Logo, os clones CL03 e CL05 são considerados benchmarks para os clones CL7 e CL01, apresentando valores de $94,21 \%$ e $45,22 \%$.

O clone CL03 apresenta-se como único clone considerado benchmark para ambas as Idades de corte avaliadas. Lado outro, verifica-se que o clone CL08 é o único clone que não é considerado benchmark para ambas as idades de corte, visto que seus valores de referência apresentam-se muito abaixo dos demais clones benchmarks.

\section{Conclusões}

Os clones CL02, CL03, CL05 e CL08 foram identificados como os clones mais eficientes em ambas as idades de corte, caracterizando-os como possíveis benchmarks para os clones considerados ineficientes. Entretanto, o clone CL04 foi considerado o único que deverá ser eliminado da lista de clones a serem utilizados nos próximos ciclos de plantio. Isso pode ser definido em função da diminuição de seu volume individual, bem como de sua densidade básica, o que ocorre aumentando sua idade de corte.

A metodologia não-paramétrica DEA pode ser considerada como uma opção para o cálculo de eficiências técnicas de clones de eucalipto, de maneira a determinar quais clones de eucaliptos são os mais eficientes e também para verificação de sua evolução com relação a sua Idade de corte.

Também foi possível identificar e comparar entre os clones classificados como ineficientes 
junto aos seus benchmarks, proporcionando uma análise mais apurada sobre quais variáveis deverão ser incrementadas e/ou reduzidas, propondo metas com vistas a buscar a sua eficiência técnica, maximizando seus volumes produzidos e minimizando seus custos.

Esses modelos ajudarão os gestores na tomada de decisão para a definição dos clones a serem eliminados dos próximos ciclos e na manutenção de sua utilização. Também foi possível definir, através de resultados dos modelos DEA, qual será a Idade de corte mais vantajosa para um determinado tipo de clone, comparando-os ao longo de suas idades de corte e possibilitando a redução na sua idade e também de seus custos.

Essas análises também poderão ser utilizadas como referência para estudos futuros, de maneira a buscar melhorias para os clones classificados como ineficientes, pela sua comparação junto aos clones classificados como eficientes.

\section{Agradecimentos}

Ao amigo Engenheiro Florestal Robson Oliveira Laprovitera (in memorian).

Ao grupo de pesquisa CENPRO - Centro de Pesquisa em Engenharia de Produção da FCA/UNICAMP.

\section{Referências}

ARAÚJO, P. M. de Q.; CARMONA, C. U. D. M. Eficiência de uma rede de agências bancárias utilizando o modelo Data EnvelopmentAnalysis - DEA. RevistaProdução, Florianópolis, v. 2, p. $1-25,2002$.

BANKER, R. D.; CHARNES, A.; COOPER, W. W. Some Models for Estimating Technical and Scale Inefficiencies in Data Envelopment Analysis. Management Science, Catonsville, v. 30, n. 9, p. 1078-1092, sep. 1984.

BARROS, T. D. et al. Avaliação dos atrasos em transporte aéreo com um modelo DEA. Production, Niterói, v. 20, n. 4, p. 601-611, dez. 2010.

CHARNES, A.; COOPER, W. W.; RHODES, E. Measuring the efficiency of decision making units. European Journal of Operational Research, [s. l.], v. 2, n. 6, p. 429-444, 1978.

CORREIA, T. C. V. D.; MELlO, J. C. C. B. S.; MEZA, L. A. Eficiência técnica das companhias aéreas brasileiras: um estudo com análise envoltória de dados e conjuntos nebulosos. Production, Niterói, v. 21, n. 4, p. 676-683, 2011.

DYSON, R. G. et al. Pitfalls and protocols in DEA. European Journal of Operational Research, [s. l.], v. 132, n. 2, p. 245-259, 2001.

FERREIRA, C. M. C.; GOMES, A. P. Introdução à análise de envoltória de dados: teoria, modelos e aplicações. Viçosa, MG: Editora UFV, 2009. 389 p.

FERREIRA, D. H. A. A. et al. Crescimento de clone de Eucalyptusurophylla x E. grandis em diferentes espaçamentos. Floresta, Curitiba, v. 44, n. 3, p. 431-440, 2014.

GOMES, E. G.; MANGABEIRA, J. A. D. C. Uso de análise de envoltória de dados em agricultura: o caso de Holambra. Revista Engevista, Niterói, v. 6, n. 1, p. 19-27, 2004.

GRATTAPAGLIA, D. Seleção genômica ampla: o novo paradigma. Revista Opiniões, Ribeirão Preto, p. 6-8, 2014.

INDÚSTRIA BRASILEIRA DE ÁRVORES. Relatório IBÁ 2017. Disponível em: http://iba.org/ images/shared/Biblioteca/IBA_RelatorioAnual2017.pdf. Acessado em: 15 ago. 2018.

MARTINI, A. J. O plantador de Eucalipitus: a questão da preservação florestal no Brasil e o 
resgate documental do legado de Edmundo Navarro de Andrade. 2004. Dissertação (Mestrado) - Faculdade de Filosofia, Letras e Ciências Humanas da Universidade de São Paulo, São Paulo, 2004.

MELLO, J. C. C. B. S. et al. Algoritmo de alocação de recursos discretos com análise de envoltória de dados. Pesquisa Operacional, Rio de Janeiro, v. 26, n. 2, p. 225-239, ago. 2006.

MELLO, J. C. C. B. S.; GOMES, E. G. Eficiências aeroportuárias: uma abordagem comparativa com análise de envoltória de dados. Revista de Economia e Administração, São Paulo, v. 3, n. 1, p. 15-23, 2004.

MEZA, L. A. et al. ISYDS - Integrated System for DecisionSupport (SIAD - Sistema Integrado de Apoio a Decisão): a software package for data envelopmentanalysismodel. Pesquisa Operacional, Rio de Janeiro, v. 25, n. 3, p. 493-503, 2005.

PEÑA, C. R. Um modelo de avaliação da eficiência da administração pública através do método análise envoltória de dados (DEA). Revista de Administração Contemporânea, Rio de Janeiro, v. 12, n. 1 , p. 83-106, mar. 2008.

SANTOS, R. B. N. dos. Eficiência técnica na indústria de base florestal brasileira via metas intermediárias. Revista Árvore, Viçosa, MG, v. 35, n. 6, p. 1319-1326, 2011.

SENRA, L. F. A. C. et al. Estudo sobre métodos de seleção de variáveis em DEA. Pesquisa Operacional, Rio de Janeiro, v. 27, n. 2, p. 191-207, ago. 2007.

SILVA, M. G. Produtividade, idade e qualidade da madeira de Eucalyptus destinada à produção de polpa celulósica branqueada. 2011. Dissertação (Mestrado) - Escola Superior de Agricultura "Luiz de Queiroz", Piracicaba, 2011.

SILVEIRA, J. Q.; MEZA, L. A.; MELLO, J. C. C. B. S. Identificação de benchmarks e antibenchmarks para companhias aéreas usando modelos DEA e fronteira invertida. Production, Niterói, v. 22, n. 4, p. 788-795, dez. 2012.

TITKO, J.; STANKEVIČIENĖ, J.; LĀCE, N. Measuring bank efficiency: DEA application. Technological and Economic Development of Economy, Italy, v. 20, n. 4, p. 739-757, 2 oct. 2014.

VILELA, D. L.; NAGANO, M. S.; MERLO, E. M. Aplicação da análise envoltória de dados em cooperativas de crédito rural. Revista de Administração Contemporânea, Rio de Janeiro, v. 11, nesp 2, p. 99-120, 2007.

WILCKEN, C. F. et al. Guia prático de manejo de plantações de eucalipto. Botucatu: [s. n.], 2008.

YANG, X. et al. Effect of Eucalyptus forests on understory vegetation and soil quality. Journal of Soils and Sediments, [s. l.] v. 17, n. 9, p. 2383-2389, 15 sep. 2017. 